Brodsky, S.L., Wilson, J.K., \& Neal, T.M.S. (2013). Refusing and withdrawing from forensic evaluations. Journal of Forensic Psychology Practice, 13, 14-26. doi: 10.1080/15228932.2013.746908

This is a pre-print version of the paper that is available on the Taylor and Francis website and at doi: 10.1080/15228932.2013.746908. This article may not exactly replicate the final version published in the journal. It is not the copy of record.

(C) 2013 Taylor and Francis 
Refusing and Withdrawing from Forensic Evaluations

Stanley L. Brodsky, Jennifer Kelly Wilson, and Tess M. S. Neal

Department of Psychology

The University of Alabama

Author Note

Tess M.S. Neal is now at the Department of Psychiatry, University of Massachusetts Medical School.

Correspondence concerning this article should be addressed to Stanley L. Brodsky, Department of Psychology, The University of Alabama, Box 870348, Tuscaloosa, AL 354870348. E-mail: sbrodsky@bama.ua.edu 


\begin{abstract}
Aside from an article by Gutheil, Bursztajn, Hilliard, and Brodsky (2004), scant literature exists regarding why forensic mental health professionals refuse or withdraw from cases. The current study collected descriptive information about the reasons mental health experts decline or withdraw from forensic assessments, both early and late in the legal process. In response to an online survey, 29 practicing forensic psychologists and psychiatrists presented examples of case withdrawal from their professional experiences. Their major reasons included ethical issues or conflicts, payment difficulties, and interpersonal or procedural problems with retaining counsel or evaluees. The results indicate that there are compelling personal and professional reasons that prompt forensic mental health experts to withdraw from or turn down cases.
\end{abstract}

Keywords: forensic evaluations, forensic psychologists, forensic psychiatrists, professional issues; case withdrawal 


\section{Refusing and Withdrawing from Forensic Evaluations}

Forensic mental health experts face a number of professional challenges during the course of their work, including potential problems with the courts and attorneys, issues with clients, personal and logistical obstacles, and ethical questions. Professional challenges may reflect a trilemma of sorts, where the expert must manage the demands of the court, the attorneys, and their own obligation to appropriate and ethical behavior. Mental health experts seek to assist the trier of fact by providing an objective assessment of the evaluee. In addition, the experts work with attorneys in providing the particular evaluation, working within a time frame, possibly testifying, and being candid regarding assessment findings (Gutheil, 2009). Last but not least, when pondering professional predicaments as they occur, forensic evaluators must consider their professional codes of ethics, ability to tolerate the demands of the situation, obstacles to conduct the evaluation in an objective manner, and levels of personal stress.

During the span of forensic experts’ careers, they may face the question of whether to decline an evaluation in the early stages or withdraw later from a case. There can be compelling reasons for forensic mental health experts to withdraw, even during late phases of a case. A modest literature exists on withdrawal of forensic experts. Gutheil, Commons, and Miller (2001) conducted a survey of 37 forensic psychiatrists regarding a number of professional issues, including attorney pressures on the expert. A subset of the survey data concerned reasons why psychiatrists had withdrawn late from forensic assessments. In a subsequent article based on the same data by Gutheil, Bursztajn, Hilliard, and Brodsky (2004), five major reasons were identified for justified withdrawal of forensic psychiatrists. These reasons included loss of objectivity, payment problems, withholding of critical data by attorneys, attorneys' failures to meet the conditions needed for an opinion, and last-minute change of focus or task. Loss of 
objectivity was described in terms of personal conflicts, conflicts of interest, or questions of possible bias. In addition to these specific reasons for withdrawal, Gutheil et al. (2004) identified three overriding motives for withdrawal as avoiding harm to reputation, eschewing the compromise of honesty and objectivity, and abstaining from potentially misleading testimony without an adequate factual basis.

We were motivated to research the topic of withdrawals due to our own professional experiences. One instance of withdrawal from a forensic case resulted from working with an incompetent attorney whose professional errors were not tolerable. In a second instance, we learned that the opposing attorneys in the case were actively spying, as they indicated through retaining counsel that they knew exactly where we were at different times of the day.

\section{Purpose}

The goal of the current study was to build on the Gutheil et al. (2004) article and to compile more information about how and why forensic mental health experts withdraw from commitments to conduct assessment of criminal or civil litigants. As noted, limited knowledge exists on reasons why mental health professionals may withdraw from forensic assessments. Our preliminary study differed from the Gutheil study in that participants included forensic psychologists as well as psychiatrists, and our study did not limit responses to withdrawals conducted late in the process of the evaluation.

\section{Method}

Forensic psychologists and psychiatrists were contacted and invited to participate in an online survey consisting of one open-ended question (see Appendix). This question inquired about reasons for and methods by which these practicing professionals had withdrawn from forensic assessment cases over their career. 
Forensic experts were contacted through one or more of three waves of data collection. The first wave consisted of the primary investigator emailing 10 forensic colleagues in his geographical region. In the second wave of data collection, approximately 100 additional forensic psychologists and psychiatrists were contacted through email and invited to participate in the survey. The third wave occurred through a forensic psychology listserv with an invitation to participate in this study. The listserv was estimated to have approximately 1000 subscribers. The survey distribution yielded 29 completed responses, most of which came from the two personal emails. This is a low response rate. The survey did request responses about experiences that likely have a low base-rate of occurring. For instance, the first author has withdrawn from cases twice in a career spanning four decades of part-time forensic assessment. We did not keep a record of names of people who replied. All participants were believed to have obtained doctoral or medical degrees. Participants received no compensation for participation.

An open-ended survey was developed on Survey Monkey and the link was sent to all prospective participants. The survey invitation simply outlined the aim as an inquiry into why forensic mental health professionals withdraw from assessment cases. Respondents were given the options to respond anonymously or to provide their name for identification in any write-up. No demographic information was collected from participants, because we were primarily seeking descriptive information about case withdrawal experiences. Participation was voluntary in that individuals had to take the affirmative action of clicking the survey link and composing and sending their reply.

\section{Results}

The content of the responses reflected the breadth of specializations in the field: criminal cases, civil cases, family and custody issues, and industrial/organizational work. Most 
respondents described multiple instances of withdrawal from forensic assessments. In addition, some participant responses denoted more than one reason for withdrawal from a single assessment. For example, scheduling problems were reported along with prior knowledge of a difficult client as occurring within a single case. Because multiple reasons for withdrawal were provided in several individual cases, many responses were represented by more than one category. After a review of the survey responses, reasons for withdrawal from forensic assessments were divided into the following categories: ethical conflicts, problems with the retaining attorney(s), trouble with evaluee, payment issues, and miscellaneous issues. Table 1 provides an overview of categories that were developed and the percentage of responses in each category.

\section{[Insert Table 1 about here]}

\section{Ethical Conflicts}

Approximately $48 \%$ of the survey responses could be placed within this category. Some overlap occurred between ethical conflicts and problems with retaining counsel, as attorneys asked forensic examiners to engage in possibly unethical activity. The various quotes in this report provide examples of these ethical problems.

Ethical concerns were evident in cases in which the forensic evaluator witnessed questionable behavior or demands by the attorney or by the evaluee. One participant stated that he or she opted to withdraw because, "the attorney that retained me asked me to perform tasks which I thought were unethical.” Some attorneys utilize various manipulative behaviors to transform or create a forensic evaluation that will be favorable for the attorney’s case; indeed, some attorneys may perceive that doing so is necessary within the adversarial environment. The evaluees may also, out of concern for their legal situation, ask or otherwise manipulate the 
forensic expert to report only information that is helpful to their case. One survey participant reported asking the court for permission to withdraw in a custody case in which the angry parent filed a complaint with the state licensing board (which ultimately was found to be baseless). Perhaps all forensic experts should opt to withdraw from assessments associated with licensing board complaints.

The majority of ethical conflicts concerned conflicts of interest that necessitated an early refusal to take the case. In a number of instances, forensic experts reported withdrawing from assessments due to prior experience as a treatment provider to the evaluee. Withdrawal in one case occurred when the evaluator realized she or he knew the evaluee from church. The respondent explained the reasoning behind this decision; "I believed that ethically I could not provide an unbiased evaluation under these circumstances. I also thought it best to withdraw before gathering information that would no doubt have made us both uncomfortable had we run into each other at church.” Another forensic expert withdrew from a case after discovering that he or she had already evaluated the co-defendant at an earlier point. Experts' withdrawal also occurred in cases in which requests from both prosecution and defense were made for the same case and when a business partner was retained by opposing counsel.

\section{Problems with Retaining Attorney(s)}

More than half (52\%) of the participant responses described problems in dealing with the retaining attorney. A forensic expert may find it difficult to work with attorneys, for instance, when defense attorneys exhibit carelessness or actions that put their client, the defendant, at unusual risk for conviction in capital trials. Other attorney carelessness identified by forensic experts included arriving late for meetings or neglecting to meet at all, not returning the expert's phone calls or emails, and general lack of preparation for trial. A forensic expert may have the 
option to withdraw from working with unprofessional attorneys, but, as one respondent wrote, "what about the poor defendants who get stuck with him?” One expert reconsidered work with an attorney who failed to protect a forensic expert from prolonged personal attacks by crossexamining attorneys.

The retaining attorney's abrasive personality was also an adequate reason for a forensic professional to avoid business with that person. This participant wrote, "the attorneys are crazy -defined variously as harassing, intimidating, argumentative, rude, combative and just plain mean. Why should I subject myself to that? There is no amount of money I can be given to put up with an attorney or a client who demands an outcome or else.” Another respondent withdrew from forensic cases in which, "the client is too difficult to manage and it starts to interfere with my other work."

Significant differences of opinion may also mar the relationship between the forensic expert and the retaining attorney. Opposing views may include such concerns as the minimum amount of data needed to conduct a thorough evaluation as well as the final conclusion regarding such evidence. One expert included among a list of reasons for withdrawing as being overall, “disagreement about what was needed to perform an adequate assessment (I recommended more; the lawyer wanted much less than I thought was necessary to establish an adequate database).” One forensic psychologist described a situation in which the middle man for the corporate client provided very limited information regarding the employee and wanted only a "quick and dirty" evaluation that answered circumscribed questions minimally and without elaboration. Other survey participants described instances where they withdrew from cases because they were ultimately unable to provide the opinion desired by the attorney. 
Differences of opinion may also suggest unethical behavior by the retaining attorney. Interpersonal manipulation may begin in the early stages of the work. As described by one participant, “There is a ‘courtship’ period where attorneys try to solicit some general thoughts about the case and if an expert's perspective is in alignment with the theory of the case. The bulk of times I withdraw it is in this initial period where I haven't been officially retained or declared, but I get the feeling I'm being lobbied. I'll often say 'thanks, but no thanks’ at this stage.” Another expert related working with a plaintiff's attorney in an international civil suit against a large organization where the plaintiffs expected to win damages in millions of dollars. After hearing that the expert would not provide a supporting opinion, the attorney urged the expert to accept a first-class trip to an exotic and beautiful part of the world. The expert declined and withdrew from the case, wishing to avoid being perceived as a "hired gun.”

Some attorneys have sought to change the report itself or create their expert's opinion. One participant reported withdrawing late when "attorneys attempted to shift my report content" in a multimillion-dollar will contest. The same expert also withdrew from a case in which, "the attorneys had prepared an affidavit based entirely on my off-the-cuff remarks on the phone to an attorney, before any retention had been acted on or any records reviewed.”

\section{Trouble with the Evaluee}

Twenty-eight percent of the responses reported various problems with the person to be assessed as the reason for withdrawal. The problems ranged from relatively benign issues, like difficulty scheduling appointments with the evaluee, to moderately serious issues like language barriers requiring interpreters, to circumstances involving explicit threats of violence. Custody battles and family court issues, for example, provided many instances of working with difficult and belligerent evaluees. One custody case resulted in death threats toward the court-appointed 
expert, who eventually requested to withdraw. Another respondent wrote, "I have withdrawn in response to threats, veiled and otherwise. I had one client who threw a concrete block through my office window. Yes, she was charged, but only with criminal mischief. She did lose custody because she had made her 7-year-old daughter lodge false sexual abuse claims against the father.” In another custody case, a forensic expert withdrew after experiencing multiple harassing phone calls and demands from one parent.

Evaluations in criminal cases also provide challenges, demands, and threats. One expert reported a case in which, "the defendant's statements about the crime story [were] very hard to believe even after challenging the defendant about this.” The evaluator opted to withdraw from the case "rather than risk testimony that would further damage the defendant." Another survey participant reported a case of civil commitment for a sexually violent person. The evaluee wrote letters to the forensic expert indicating what to write in the report and even included a completed PCL-R with instructions for the expert on how to score the measure. This all occurred before the expert had any meetings with the evaluee. The evaluator felt that his/her "ability to do an objective evaluation to my standards was compromised,” and elected to withdraw.

\section{Payment Issues}

Issues with payment occupied almost one quarter (24\%) of the survey responses. These responses simply mentioned that lack of payment led to withdrawal, often a failure to pay the initial retainer or failure to replenish the retainer funds. A few responses provided more specific examples of payment-related problems leading to withdrawal. One professional related discontinuing a case due to the judge’s “unrealistic cap on fees.” The prospect of no payment was also mentioned, as one expert withdrew after being asked to evaluate pro bono an entire family in a custody suit. The participant explained her reasoning: "Custody work is too 
dangerous for me to have given at least a hundred hours of my time and effort for no compensation.” Lastly, a respondent reported withdrawing after learning of a colleague’s bad experience with a government agency. This potential client - the agency - not only failed to pay in a timely manner but also "nickel and dimed" the colleague over minor expenses. The respondent concluded that, "her experience was so horrid on so many levels that I did not want to go through it myself.”

\section{Miscellaneous Issues}

This catchall category represents $31 \%$ of the survey responses. Beyond problems with attorneys and evaluees, some experts described troubles with certain judges and opposing experts. Issues with judges concerned limits on fees that the expert could charge, a requirement that a neuropsychological evaluation be videotaped, and a judge’s troublesome interpersonal behavior. A few respondents reported having issues with the opposing expert; one psychologist explored the nature of these issues in detail. This family law expert withdrew from cases multiple times due to the prospect of testifying opposite a "rogue" expert who had no experience in the specific area of expertise.

Other participant responses included one participant who reported having difficulty negotiating appointment times with staff at the jail in order to evaluate the defendant. The retaining attorney did not assist with any scheduling negotiations, and the expert eventually withdrew. More than one forensic expert noted the difficulties of working in contentious environments, especially in family law and custody cases. As one participant wrote "few practitioners are willing to work in this minefield.” One court-appointed expert noted withdrawing due to the level of animosity and the accusations of partiality by attorneys on both sides. 


\section{Difficult Decisions}

Survey participants sometimes described how they decided to withdraw from cases, clearly considering the option to withdraw very seriously. Experts often described their motivations in terms of protecting their own mental health (avoiding stress, headaches, and worry) and maintaining ethical practice standards. One mental health expert described the difficult decision in two withdrawals from capital cases; "I consulted with peers before making the decision in each case. I found the experience to be wrenching and whereas I was confident I had done the right thing in one case, I have doubts to this day about the other case and suspect I will never be comfortable about my decision.” One forensic psychologist reported discussing the option to withdraw with his graduate students in clinic supervision as a real-life example of the kinds of professional challenges that forensic experts face.

\section{The Act of Withdrawing}

Some of the forensic experts who participated in this survey described their process of withdrawing. Participants frequently notified the attorneys as soon as possible regarding the decision to withdraw. Some reported that they advised the attorneys in the initial stages the conditions under which they would withdraw from a case (e.g., if the judge requires videotaping of the neuropsychological testing). In several examples, the experts needed to formally request withdrawal from the judge, providing valid reasons why a presumably late withdrawal was necessary (e.g., accusations of bias, threats or acts of physical harm by the evaluee).

In some cases, the forensic experts explained thoroughly to retaining parties their reasons for discontinuing the assessment. In other instances, however, the expert felt for various reasons the need to limit the explanation. In the case of difficult or harassing clients, one participant described a polite approach to withdrawing; "I never tell them they are crazy, of course. I simply 
state that I am unable to meet their needs and I return the money if pre-paid. I call and I follow up with a letter.” This expert also described a straightforward style of discontinuation when the client has not paid the retainer fee at the time of meeting; "I will be happy to reschedule when you can get the money.” In response to attorneys' attempt to change the content of a forensic report, one expert reported the late withdrawal thusly, "I sent them a letter explaining why, with the specific line authorizing this highlighted from a copy of my contract. I sent back the entire database (large crate) and told them never to call me again.” The same forensic expert also experienced an instance of clearly unacceptable attorney behavior, which prompted serious discussion with legal consultants about “misappropriation of a likeness” and resulted in a journal article about the experience. On some occasions, withdrawing experts offer the names of other forensic professionals to the attorney. One respondent wrote about doing this and reported that a “highly qualified friend,” perhaps “seduced by the glitz of the case,” succumbed to the attorney’s venal pressures.

\section{Discussion}

We collected descriptive information about the reasons psychologists decline or withdraw from forensic assessments. In response to this online survey, practicing forensic mental health experts presented examples from their professional experiences. Their major reasons for withdrawal included ethical issues or conflicts, payment difficulties, and interpersonal or procedural problems with retaining counsel or evaluees. The results indicate that there are cogent and compelling personal and professional reasons that prompt mental health professionals to withdraw from commitments to forensic cases. Our survey results echo some of Gutheil et al.’s (2004) results as well as other literature discussing problems encountered by forensic mental health practitioners. 
Ethical concerns certainly governed some of the withdrawals reported by experts. Gutheil (2009) asserted that, in order to properly orient one’s “ethical compass” (p. 8), an expert must have the ability to turn away cases and not feel constrained to accept every evaluation. The expert's turndown rate might then be conceptualized as an indirect measure of ethical behavior. In the same manner, the forensic professional must maintain the ability to withdraw from assessments where ethical values become compromised. As Appelbaum (1997) advised regarding such predicaments, the expert should walk away from a case rather than sacrifice ethical principles. Maintaining personal and professional ethical values assists the forensic practitioner in being an "honest expert” for the courts (Gutheil, 2009, pp. 15).

The Gutheil et al. (2004) study found some respondents who attributed their late withdrawals to a severe loss of objectivity. Our findings provided similar examples, from simple conflicts of interest to unethical behavior by attorneys and/or evaluees. The range of conflicts found in the present survey reflects a number of different motives. For example, respondents who reported turning down a case due to a prior therapeutic relationship were acting in accord with ethical standards prohibiting treatment providers from engaging in forensic assessment of therapy clients (Strasburger, Gutheil, \& Brodsky, 1997). In addition, given the helping ethic that is common to training in psychology and psychiatry, Gutheil and Simon (2004) referred to the clinicians’ tendency to view the evaluee empathically as a therapy patient a “treater bias” (pp. 261).

Some of the experts' ethical conflicts concerned behavior by the attorneys who retained them. Similar to the descriptions of unprofessional or irresponsible attorneys reported here, Strasburger, Miller, Commons, Gutheil, and LaLlave (2003) found that 39\% of surveyed forensic psychiatrists rated as highly or extremely stressful the prospect of an unprepared retaining 
attorney. A few of Strasburger and colleagues’ survey responses also observed withholding of relevant data by attorneys, which was reported in the present study as well. Gutheil et al. (2004) reported that $49 \%$ of their respondents acknowledged that their retaining attorneys withheld data that were sometimes critical, a behavior that factored heavily into experts' decisions to withdraw late in the process.

As discovered in the present survey regarding withdrawal from assessments, attorneys may attempt to influence the forensic mental health expert beyond simply withholding certain records from review. Tactics by the retaining client may take the form of subtle or overt seduction. As already noted, one respondent in the current study reported resisting an attorney’s attempts to influence the expert's opinion through the promise of a trip to an exotic locale. The present study included experts’ descriptions of other attorney attempts to influence, through "lobbying” for a preliminary opinion and arguing regarding the conclusion.

Beyond withholding data, financially seducing, or even threatening the expert, attorneys may attempt to change the expert's conclusion or fabricate the expert opinion in its entirety. Our survey data included a few examples of this behavior, which incited participants to withdraw from the business relationship. Money matters were some experts’ justification for withdrawing from cases.

In addition to various issues with attorneys, forensic mental health experts could experience any number of different problems with the evaluee. The present study included examples of scheduling issues, a less-than-credible evaluee, language barriers, evaluee attempts to influence the expert opinion, and even harassment and threats. In our experience, as well, some forensic mental health professionals are harassed or threatened and eventually retain their own attorneys or obtain unlisted phone numbers. Not surprisingly, forensic practitioners may 
aim to avoid this occupational hazard as soon as red flags for such threatening behavior appear, by refusing or withdrawing from certain cases. For example, Sadoff (2011) illustrated a professional experience in which an evaluee, who normally carried a gun with him at all times, refused to leave his gun at home to attend the evaluation. After several unsuccessful attempts to convince the evaluee and forewarning that the expert would not continue otherwise, the expert halted the examination and withdrew from the domestic relations case.

The described problems provide ample cause for stress in the forensic mental health expert. Some were seriously concerned by the threats of harm directed at them. A number of our respondents reported the most contentious work to lie within child custody and family law, and experts appointed by the court were often the recipient of harassment by both sides in the case. It is clear to us that it is sufficient justification for experts to withdraw to avoid harm.

This study described the conflicts and problems faced by mental health experts in their dealings with attorneys and the courts. The detailed description of experts' experiences may assist those who are new to the profession and naïve to attorney manipulations. Some forensic mental health experts may choose to re-evaluate their personal and professional standards, threshold of stress, and the content of written business contracts with future clients.

The methodology of the present study has some limitations. The use of a survey necessarily limited the amount of information gathered. Survey respondents also may differ from non-respondents in various and unpredictable ways. Participants were gathered as a convenience sample and do not necessarily reflect a random or representative sample.

The open-ended nature of this study may be a foundation for those who conduct future research on related professional issues for forensic mental health professionals. The detailed 
descriptions of experiences provide a basis for examination of the dynamics inherent in interactions with attorneys, opposing experts, evaluees, judges, and the court system. 


\section{References}

Appelbaum, P. S. (1997). A theory of ethics for forensic psychiatry. Journal of the American Academy of Psychiatry and Law, 25, 233-247.

Gutheil, T. G. (2009). The psychiatrist as expert witness ( $2^{\text {nd }}$ ed.). Arlington, VA: American Psychiatric Publishing.

Gutheil, T. G., Bursztajn, H., Hilliard, J. T., \& Brodsky, A. (2004). “Just say no”: Experts’ late withdrawal from cases to preserve independence and objectivity. Journal of the American Academy of Psychiatry and Law, 32, 390-394.

Gutheil, T. G., Commons, M. L., \& Miller, P. M. (2001). Withholding, seducing, and threatening: A pilot study of further attorney pressures on expert witnesses. Journal of the American Academy of Psychiatry and Law, 29, 336-339.

Gutheil, T. G., \& Simon, R. I. (2004). Avoiding bias in expert testimony. Psychiatric Annals, 34, 260-270.

Sadoff, R. L. (2011). Ethical issues in forensic psychiatry: Minimizing harm. West Sussex, UK: Wiley-Blackwell.

Strasburger, L.H., Gutheil, T. G., \& Brodsky, A. (1997). On wearing two hats: Role conflict in serving as both psychotherapist and expert witness. American Journal of Psychiatry, 154, 448-456.

Strasburger, L.H., Miller, P. M., Commons, M. L., Gutheil, T. G., \& LaLlave, J. (2003). Stress and the forensic psychiatrist: A pilot study. Journal of the American Academy of Psychiatry and Law, 31, 18-26. 
Table 1.

Categories and Percentages of Reasons for Withdrawal

Percentage of Responses

Ethical Conflicts. $.48 \%$

Questionable behavior by attorney or evaluee

Conflicts of interest (e.g., previous relationship with defendant)

Problems with Retaining Attorney(s) $.52 \%$

Unprofessional or irresponsible attorney behavior (e.g., extreme carelessness, repeated failure to return phone calls, lack of preparation for trial, pattern of failure to protect expert during cross-examination)

Differences of opinion regarding conclusion

Attorney personality issues

Withholding of data (e.g., providing very limited information and asking for a "quick and dirty" evaluation)

Purpose of evaluation changed

Attempts to influence the expert (e.g., being offered an expensive vacation)

Changing or creating the expert's opinion

Trouble with Evaluee $.28 \%$

Threats or harassment by evaluee (e.g., having a brick thrown through window)

Evaluee not credible

Evaluee attempts to influence expert

Language barrier with evaluee

Payment Issues.

Lack of payment

Unrealistically low compensation

Miscellaneous.

Problems with the judge

Problems with the opposing expert (e.g., testifying opposite a "rogue" expert with no experience in the area)

Scheduling issues (e.g., negotiating appointment times at jail with staff or non-responsive attorneys)

Highly contentious environment

Accusations of bias

Note. $N=29$. Many participant responses were coded in more than one category. 


\section{Appendix}

\section{Survey Question}

I am conducting a study of why forensic mental health professionals withdraw from assessment cases. There seems to be little knowledge about this topic. If you have withdrawn from cases, I would appreciate it if you would click the link below to describe what happened and what prompted you to withdraw. Let me know in your response if you wish to be identified in any write-up. Otherwise, I will assume you wish to keep your identity private. The responses will be summarized for a report, and some illustrative descriptions will be included. Please consider any participation to be voluntary, and write if the topic interests you or if the exercise itself appears to be interesting. 